\title{
CAMERA CALIBRATION WITH IRRATIONAL RADIAL DISTORTION MODEL WITH ANALYTICAL SOLUTIONS.
}

\author{
${ }^{1}$ G.B. Ikokou, ${ }^{2}$ J. Smit \\ ${ }^{1}$ Geomatics Department, Tshwane University of technology, Pretoria, South Africa,ikokougb@tut.ac.za \\ ${ }^{2}$ Geomatics Division, University of Cape Town, South Africa,Julian.smit@uct.ac.za
}

KEY WORDS: Lens distortions, Barrel distortion, Pincushion distortion, Irrational distortion model

\begin{abstract}
ABSTARCT
Consumer grade digital cameras are widely used in many applications including Photogrammetric mapping and 3D modelling. One common limitation found in such cameras is radial lens distortions. To produce wide angle lenses camera manufacturers reduce the amount of barrel distortion by minimizing both the central and edge distortion profiles, resulting in a mixture of pincushion and barrel distortions for a single lens. These lenses also lack symmetry, making some of the existing distortion models almost ineffective. The mostly used model for radial distortion corrections is the polynomial model which is difficult to solve analytically especially when the model possesses many quadratic terms. Suggestions were made for division models but such models are not suitable when the lens field of view exceeds 180 degrees and exhibit some instabilities when dealing with large magnitude of distortion coefficients. Moreover mathematical formulations of some models cannot handle negative distortion coefficients. Attempts to improve the division models were made with proposals for rational models which present the advantage of handling larger distortion magnitude with fewer terms. However some of these models do not account for all the distortion coefficients in their solutions, limiting the potential of the techniques. This study presents an irrational distortion model with analytical solutions. The proposed model was tested with imagery captured by wide angle lenses and the experimental results reveal that the technique produced the best estimates of radial distortion coefficients. The proposed model was also able to capture image distortions originating from projection errors by the wide angles lenses used in this study.
\end{abstract}

\section{INTRODUCTION}

The mass production of consumer grade digital cameras has resulted in their integration in the Photogrammetry production environment. However a large number of those cameras are not perfect and tend to show variety of aberrations (Shah and Aggarwal, 1996). These aberrations mostly originate from off-the shelf lenses that exhibit a substantial amount of distortion. In fact, these lenses have limited field of view and increase of field of view by lens manufacturers can induce undesired effects on the image (Tommaselli et al., 2014). For instance to have wide angle lenses suitable for mapping applications the lens manufacturers reduce the amount of barrel distortions by minimizing both central and edge distortion profiles, resulting in mixture of pincushion and barrel distortions in a single lens, which are more difficult to model. Another alternative is to create a panorama image from multiple cameras but the challenge with such technique is that the final image contains heterogeneous distortion profiles originating from individual lenses and requires a very large amount of points to solve the distortion parameters and rectify the imagery (Tagoe et al., 2014). Additionally, these lenses also lack symmetry. These limitations make the need for camera calibration very important and probably more challenging to perform. Among the main aberrations produced by off-the shelf lenses is the radial distortions. There exists three types of radial distortions namely barrel, pincushion and 'moustache' distortions. In the occurrence of barrel distortions image magnification decreases when moving away from the optical axis, giving an appearance that the image was mapped around a sphere or barrel. Mathematically, barrel and pincushion distortion are quadratic functions, meaning that they increase as the square of the distance from the center increases. In the case of 'moustache' distortion function, the quartic or fourth degree term is said more dominant, while for barrel distortion function the second-degree term is more dominant in the center. On the other hand, the fourthdegree term was reported more dominant at the edges of the image for the pincushion profile (Walree, 2009). However, it is also possible that an image exhibits pincushion distortion in the center and barrel distortions at the edge. Attempts have been made to correct radial distortions using the polynomial model (Prescott and McLean, 2005; Wu et. al., 2017), division model (Fitzgibbon, 2001; Brauer-Burchardt and Vos, 2001) and rational models (Ma et al., 2003). The polynomial radial distortion model lack an inverse undistorted model and cannot be solved analytically while some division models can only perform well with very small magnitudes of distortion in the image and would not handle barrel distortions due to their mathematical formulation. Some inverse rational un-distortion models have the drawback of not accounting for the first coefficient of the model, limiting their performances. In this study we are proposing an irrational model which addresses some of the above limitations. The model formulation enables it to handle barrel, pincushion distortions as well as their combination in a 'moustache' profile. Moreover, the different parameters of the model can be solved analytically.

\section{RELATED WORK}

Radial distortion is the most common type of distortion encountered in Photogrammetry (Tardif et al., 2009; Hamad et al., 2017; Shih and Tung, 2017). Radial distortion alters the location of the image point inward or outward with reference to the image center. The inward displacement of the image point is generally described as a negative displacement of the image piont and is termed as barrel distortion while its opposite is described as 
pincushion distortion (Weng et al., 1992; Vass and Perlaki, 2003). The commonly adopted expression of radial distortion is given by a polynomial function as follows:

$$
r_{u}=r_{d}\left(1+k_{1} r^{2}+k_{2} r^{4}+k_{3} r^{6}+\ldots\right)
$$

With $k_{1}, k_{2}, k_{3} \ldots$ the coefficients of radial distortion and $r$ the radial distance. De Villiers (2010) pointed out that the expression in (1) is mostly dominated by the first term and any more elaboration of the model could create numerical instability. Despite its efficiency with fewer terms Drap and Lefevre (2016) reported that the model does not have an inverse equivalent and cannot be solved analytically especially when employed with more terms. The model would not be validated for values of distortion coefficients that nullify the expression in the denominator, limiting its performance. To address the above limitation Fitzgibbon (2001) proposed the division model given by the equation as follows:

$$
r_{u}=\frac{r_{d}}{1+k_{1} r^{2}}
$$

The rational model proposed by Brauer-Burchardt and Vos (2001) is a variation of the solutions in (2) given by the expression:

$$
r_{u}=r_{d} \frac{\sqrt{4 k r^{2}}-1}{2 k r}
$$

However the formulation of the model limits its performance since it makes it unstable for negative distortion coefficients and the term in the denominator is only valid for non-zero values of the distortion coefficient. The other limitation of the proposed approach is that it is difficult to solve analytically. Ma et al., (2003) proposed a family of rational models which are functions of traditional polynomial models. One advantage of the proposed techniques is that they are distorted undistorted models. However one limitation of the proposed family of models is the restriction of the magnitude for the second radial distortion coefficient $k_{2}$ as illustrated in equation (4) describing the fifth model in Ma et al.,(2003) as follows:

$$
r_{u i}=r_{d i} \frac{1+k_{1} r}{1+k_{2} r^{2}}
$$

To analytically estimate the distortion coefficients $k_{1}$ and $k_{2}$ the expression in (4) can be expanded and simplified to produce two linear equations (5) and (6) satisfying the coordinates of two image points $i$ and $j$ as follows:

$$
\begin{aligned}
& r_{d i}+r r_{d i} k_{1}=r_{u i}+r^{2} r_{u i} k_{2} \\
& r_{d j}+r r_{d j} k_{1}=r_{u j}+r^{2} r_{u j} k_{2}
\end{aligned}
$$

Isolating the coefficient $k_{2}$ from (5) and substituting it into equation (6) enables to analytically estimate $k_{1}$ as follows:

$$
k_{1}=\frac{r_{d j} r_{u i} r^{2}-r_{u j} r_{u i} r^{2}-r^{2} r_{u j} r_{d i}+r^{2} r_{u j} r_{u i}}{r^{3} r_{u j} r_{d i}-r^{3} r_{d j} r_{u i}}
$$

With knowledge of at least two image points and their undistorted coordinates the first radial distortion coefficient can be estimated and its value substituted into either (5) or (6) to analytically solve the second coefficient $k_{2}$

\section{METHODOLOGY}

\subsection{Calibration data}

The simulated near-error free image points' coordinates and the measured points' coordinates were respectively estimated and extracted from photographs of the calibration field in the Geomatics Department at University of Cape Town and presented in Tagoe et al., (2014). The near error free coordinates were determined using the collinearity equation as follows:

$$
\begin{aligned}
& x_{f}-x_{0}=-f \frac{L_{1}\left(X_{P}-X_{0}\right)+L_{2}\left(Y_{P}-Y_{0}\right)+L_{3}\left(Z_{P}-Z_{0}\right)}{L_{7}\left(X_{P}-X_{0}\right)+L_{8}\left(Y_{P}-Y_{0}\right)+L_{9}\left(Z_{P}-Z_{0}\right)} \\
& y_{f}-y_{0}=-f \frac{L_{4}\left(X_{P}-X_{0}\right)+L_{5}\left(Y_{P}-Y_{0}\right)+L_{6}\left(Z_{P}-Z_{0}\right)}{L_{7}\left(X_{P}-X_{0}\right)+L_{8}\left(Y_{P}-Y_{0}\right)+L_{9}\left(Z_{P}-Z_{0}\right)}
\end{aligned}
$$

With $x_{f}, y_{f}$ the estimates of distortion free points' coordinates, $x_{0}, y_{0}$ the coordinates of the principal point and $X_{P}, Y_{P}$ and $Z_{P}$ the coordinates of the world point in the object space coordinate system while $X_{0}, Y_{0}$ and $Z_{0}$ are the coordinates of the approximate camera position and $f$ the camera constant, $L_{1}, L_{2} \ldots L_{9}$ are the elements of the rotation matrix. The table1 presents an extract of the point data used in this experiment. From the measured and undistorted coordinates we estimated the radial distorted and undistorted radi for each couple of point as illustrated in table1.

\begin{tabular}{|l|l|l|l|l|l|l|}
\hline $\begin{array}{l}\text { Points } \\
\text { ID }\end{array}$ & $x_{u}$ & $y_{u}$ & $x_{d}$ & $y_{d}$ & $r_{u}$ & $\mathbf{r} r_{d}$ \\
\hline $\mathbf{1}$ & 59,88 & 37,98 & 59,87 & 37,94 & 2514,05 & 2511,93 \\
\hline $\mathbf{2}$ & 59,99 & 32,54 & 59,98 & 32,62 & 2328,83 & 2330,83 \\
\hline $\mathbf{3}$ & 49,45 & 38,43 & 49,63 & 38,43 & 1961,08 & 1970 \\
\hline $\mathbf{4}$ & 49,57 & 32,95 & 49,75 & 32,98 & 1771,44 & 1781,3 \\
\hline $\mathbf{5}$ & 49,37 & 28,06 & 49,57 & 28,18 & 1612,38 & 1652,65 \\
\hline $\mathbf{6}$ & $-11,51$ & 49,72 & 10,45 & 44,46 & 130,28 & 1042,95 \\
\hline $\mathbf{7}$ & $-10,63$ & 9,44 & 12,04 & 18,63 & 101,06 & 246,02 \\
\hline $\mathbf{8}$ & $-11,42$ & 60,2 & 11,54 & 50,86 & 1877,23 & 1359,96 \\
\hline $\mathbf{9}$ & 106,38 & $-5,57$ & 85,57 & 9,72 & 5673,87 & 3708,35 \\
\hline $\mathbf{1 0}$ & 120,82 & $-6,56$ & 90,26 & 11,62 & 7320,25 & 4140,95 \\
\hline
\end{tabular}

Table 1: distortion free coordinates and measured coordinates with their respective radi. 


\subsection{Distortion model}

With distortion profiles becoming more and more complex, their modelling with traditional polynomials has become almost inefficient especially when dealing with severe distortions produced by wide angle lenses such as fish eye lenses and panorama systems. The motivation on the choice of an irrational model was driven by the fact that some distortion measures cannot be expressed as rational or integer numbers thus cannot be correctly modelled by techniques relying on such formulations. Irrational functions instead have the advantage of capturing measurements not derived from a division of integers within the image (Jourdain, 1908). The model adopted in this study is a combination of two irrational functions and was formulated in such a way that strengthens the properties of each function. The original model is presented in the equation (9) as follows:

$$
r_{u k}=r_{d k} \frac{\sqrt{k_{p+1} \varepsilon_{k(u i, d i)}^{2(p+1)}+1}}{\sqrt{1+k_{p} \varepsilon_{k(u i, d i)}}}
$$

With the relationship between a distorted image point and its undistorted corresponding given by the equation (8) as follows:

$$
x_{u}=x_{m}+\delta_{d}
$$

With $x_{m}$ the measured point coordinates and $\delta_{d}$ the amount of radial distortion error estimated from equation (9) as follows:

$$
\delta_{d}=\frac{\sqrt{k_{p+1} \varepsilon_{k(u i, d i)}^{2(p+1)}+1}}{\sqrt{k_{p} \varepsilon_{k(u i, d i)}+1}}
$$

With $\varepsilon_{k}$ the radial distance between the measured and undistorted position of a point $k$ in the image. Dividing the model in (9) by $r_{d k}$ which is the radial distance from the image center to the distorted image point, we can rewrite the model as follows:

$$
\frac{r_{u k}^{2}}{r_{d k}^{2}}=\frac{k_{p+1} \varepsilon_{k(u i, d i)}^{2(p+1)}+1}{k_{p} \varepsilon_{k(u i, d i)}+1}
$$

An expansion and simplification of the model in (9) produces linear equations as functions of measured and near distortion free coordinates as follows:

$$
r_{u i}^{2} \varepsilon_{k(u i, d i)} k_{p}-k_{p+1} \varepsilon_{k(u i, d i)}^{2(p+1)} r_{d i}^{2}=r_{d}^{2}-r_{u i}^{2}
$$

It requires at least two image points with their undistorted coordinates' measurements to analytically solve the radial distortion coefficients but the use of more points would improve the accuracy of the estimated parameters. Moreover the mathematical formulation in (13) shows that the model deals with asymmetric radial distortion profiles.

\subsection{Model evaluation and discussion}

The table 2 presents the estimated distortion coefficients of four radial distortion models including the traditional two coefficients polynomial model, the single coefficient rational model, the two coefficients division model and the proposed two coefficients irrational model. The overall results show that the images used for this experiment contain the 'mustache' distortion profile characterized by opposite signs of coefficients $k_{1}$ and $k_{2}$ (Tang et al., 2017). Individual barrel and pincushion profiles were also perceived from the computed distortion coefficients and characterized by similar coefficients signs. In terms of minimizing the distortion within the image with the first radial distortion coefficient $k_{1}$ the polynomial model performed the poorest followed by the division model. The rational and the proposed model produced better results with the irrational model producing the least coefficient as illustrated in the figure 1.

\begin{tabular}{|l|l|l|l|l|}
\hline $\begin{array}{l}\text { Coefficie } \\
\text { ts }\end{array}$ & $\begin{array}{l}\text { Polynom } \\
\text { ial }\end{array}$ & Rational & Division & $\begin{array}{l}\text { Irrationa } \\
1\end{array}$ \\
\hline$k_{1}$ & $4.36 \times 10^{-2}$ & $4.85 \times 10^{-5}$ & $5.32 \times 10^{-3}$ & $3.54 \times 10^{-7}$ \\
\hline$k_{2}$ & $-4.46 \times 10^{-7}$ & -------- & $-6.98 \times 10^{--}$ & $-2.01 \times 10^{-9}$ \\
\hline
\end{tabular}

Table 1: Estimated distortion coefficients $k_{1}$ and $k_{2}$ per distortion model

When it comes to the performance of the model to handle distortion with the second radial distortion coefficient $k_{2}$ ,the division model produced the lowest coefficient followed by the polynomial and irrational models. The larger radial distortion coefficient $k_{2}$ observed with the irrational model may originate from large discrepancies between a certain number of measured points and their corresponding near error free as illustrated by the points 9 and 10 in table 1 . The discrepancies could originate from a severe projection error by the fisheye lenses used to capture the imagery. It appears that the irrational distortion model has successfully captured a magnitude of radial distortion produced by the fisheye lenses and which was beyond the measuring power of the other radial distortion models (Jourdain, 1908) as illustrated in figure2. 


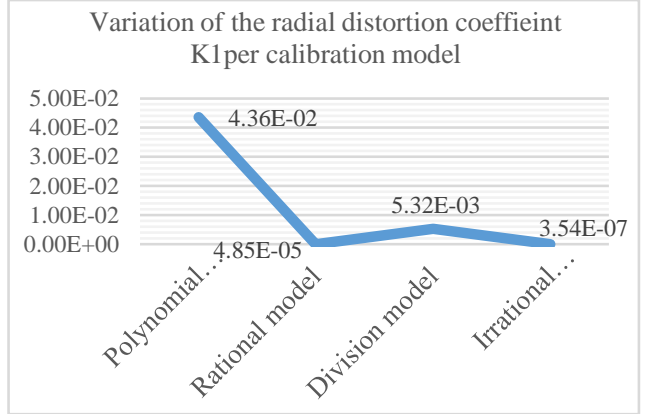

Figure1: Performance comparison of distortion models with reference to the first coefficient of radial distortion $k_{1}$.

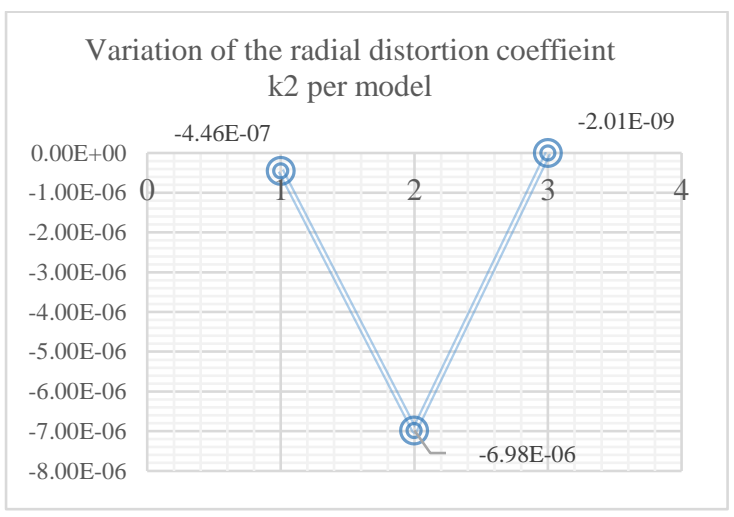

Figure 2: Performance of distortion models with reference to the second coefficient of radial distortion $k_{2}$

\section{CONCLUSION}

This study proposed a radial distortion model based on irrational function. The model formulation ensured that it can handle any signs of distortion coefficients and can handle complex distortion profiles such as mustache distortions. The model parameters can be solved analytically without any need for iteration or optimization process. The proposed model enabled to capture projection errors originating from the fish eye lenses through its second distortion coefficient $k_{2}$. This provides a unique advantage over the other methods when correcting distortions with the inverse models. The results of this study could be improved with accurate rigid body transformation results as any error in the coordinate estimation would affect the final distortion coefficients' estimates. Moreover the results could also be improved by extending the technique as a two stages calibration in which the analytically estimated distortion coefficients would be considered as initial values in an iterative process until the discrepancies between the initial values and the measured coordinates are minimized at their best. Further studies on this research would focus on extending the model by adding more distortion coefficients.

\section{REFERENCES}

Brauer-Burchardt, C., Voss K., 2001. A new algorithm to correct fish-eye- and strong wide-angle-lens-distortion from single images. In: IEEE International Conference on Image Processing. Volume 1. (2001) 225 - 228

De Villiers, J., 2010. Modeling of radial asymmetry in lens distortion facilitated by modern optimization techniques. In: Proceedings of the 2010 Electronic Imaging Conference, EI2010, vol. 10, pp. 1-8 (2010)

Drap, P., Lefevre, J., 2016. An exact formula for calculating inverse radial lens distortions. Sensors, Vol. 16(6).

Fitzgibbon, A.W., 2001. Simultaneous linear estimation of multiple view geometry and lens distortion. In: IEEE International Conference on Computer Vision and Pattern Recognition (CVPR). (2001) 125-132Tardif J.P, Sturm P., Trudeau M, and Roy S., 2009. Calibration of cameras with radially symmetric distortion. PAMI, 31(9):15521566, 2009.

Hamad R., Sattar S.A., Al-Azawi. R., 2017. Calculation the inverse radial distortion model based on Zhang method. Advances in Natural and Applied Sciences. 11(3); Pages: $86-90$

Jourdain, P.E., 1908. The introduction of irrational numbers. The Mathematical Gazette, 4(69), pp.201-209.

Ma, L., Chen Y.Q. and Moore K.L. 2003. , A new analytical radial distortion model for camera calibration. http://arxiv.org/pdf/cs/0307046.pdf, 2003.

Precott, B and McLean, G.F., 1997. Line-based correction of radial lens distortion. Graphical Models Image Process. 59(1), 39-47 (1997). 16.

Shah, S. and Aggarwal J.K., 1996. Intrinsic parameter calibration procedure for a (high distortion) fish-eye lens camera with distortion model and accuracy estimation. Pattern Recognition 29(11): 1775-1788.

Shih, M.H. and Tung, S.H., 2017. A method for correcting radial distortion based on verifying the planarity of specimens. Sadhana 42, Issue 11, pp. 1943-1952, November 2017.

Tagoe, N.D., Rüther, H. and Smit, J., 2014. A Pragmatic Approach for Lens Distortion Correction from a Fictitious Image. Proceedings of AfricaGEO 2014, pp.1-12.

Tang, Z., von Gioi, R.G., Monasse, P. and Morel, J.M., 2017. A precision analysis of camera distortion models. IEEE Transactions on Image Processing, 26(6), pp.2694-2704.

Tommaselli, A.M.G., Marcato Jr, J., Moraes, M.V.A., Silva, S.L.A. and Artero, A.O., 2014. Calibration of panoramic cameras with coded targets and a 3D calibration field. The International Archives of Photogrammetry, Remote Sensing and Spatial Information Sciences, 40(3), p.137.Wu, F., Wei, H.,

Wang, X.2017. Correction of image radial distortion based on division model. Opt. Eng. 2017, 56, 013108.

Van Walree, P., 2009. Distortion. Photographic optics. http://toothwalker. Org/optics/distortion. html, 4. 